\title{
Asymmetric Defluoroallylation of 4-Trifluoromethylpyridines Enabled by Umpolung C-F Bond Activation
}

\author{
Fei-Yu Zhou and Lei Jiao* \\ Center of Basic Molecular Science, Department of Chemistry, Tsinghua University, Beijing 10084, China \\ $C$ - $F$ bond activation, $N$-boryl pyridiyl anion, asymmetric defluoroallylation, Ir catalyzed asymmetric allylation
}

\begin{abstract}
Carbon-fluorine bond activation reaction of the trifluoromethyl group represent an important approach to fluorine-containing molecules. While selective defluorofunctionalization reactions of $\mathrm{CF}_{3}$-containing substrates have been achieved by invoking difluorocarbocation, difluorocarboradical, or difluoroorganometallic species as the key intermediate, the transformations via fluorocarbanion mechanism remained a limited success. Furthermore, the enantioselective defluorotransformation of $\mathrm{CF}_{3}$ group has not yet been realized. Herein, we report a defluorofunctionalization reaction of 4trifluoromethylpyridines involving pyridyldifluoromethyl anion as the key intermediate, which was developed based upon our previous studies on the $\mathrm{N}$-boryl pyridyl anion chemistry. When combined with Ir-catalysis, asymmetric defluoroallylation of 4-trifluoromethylpyridines could be achieved to forge a difluoroalkyl-substituted chiral center. The present work opens up a new opportunity for the defluorofunctionalization of $\mathrm{CF}_{3}$ group, and provides new insights into the $\mathrm{N}$-boryl pyridyl anion chemistry.
\end{abstract}

Fluorine-containing compounds usually exhibit unique properties $^{1}$ (e.g., m.p., b.p., pKa, lipophilicity, etc.) and are widely used in pharmaceuticals and agrochemicals. ${ }^{2}$ Among them, the molecules bearing fluoroalkyl motifs constitute a major category, which have attracted a broad research interest and thus enhanced the demand for efficient synthetic protocols. ${ }^{3}$ In this line, a well-established approach is the introduction of a fluoroalkyl group into a substrate molecule, referred to as the fluoroalkylation strategy, which is empowered by a series of fluoroalkylating reagents. ${ }^{4}$ On the other hand, the modification of a preexisting fluoroalkyl group in a molecule by $\mathrm{C}-\mathrm{F}$ bond activation represents a more flexible yet less developed strategy. ${ }^{5}$ In particular, the defluorofunctionalization of a trifluoromethyl group has been attracting an increasing attention, ${ }^{6}$ due to its common existence in fluorinecontaining compounds and the challenge associated with the activation of its $\mathrm{C}-\mathrm{F}$ bond.

Recently, a series of $\mathrm{CF}_{3}$ defluorofunctionalization reactions have been developed, with different difluorocarbon species as the key intermediate (Scheme 1). Lewis-acid was found to promote difluorocarbocation formation from simple trifuromethyl arenes, and subsequent nucleophilic substitution enabled the monoselective C-F functionalization (Scheme 1A). ${ }^{7}$ Single electron transfer (SET) could induce the $\mathrm{C}-\mathrm{F}$ bond cleavage in $\mathrm{CF}_{3}$ to afford difluorocarboradicals, enabling radical-type defluorofunctionalization (Scheme 1B). ${ }^{8}$ Recently, Wang and Houk discovered that boron radical participated in the formation of difluorocarboradical species from trifluoromethylcarbonyl compounds via a spin-center shift (SCS) process, which allowed for progressive multiple C-F bond activation (Scheme 1C). ${ }^{9}$ Transition-metal catalysis has also been involved in defluorofunctionalization of the $\mathrm{CF}_{3}$ group. Palladium could undergo a formal C-F bond oxidative addition with trifluoroarenes to generate aryldifluoromethylpalladium species amenable for cross-coupling, as reported by the Zhang group (Scheme 1D). ${ }^{10}$ Very recently, the Bi group demonstrated that the in-situ generated rhodium trifluoromethylcarbenoid was able to produce difluoromethyl ketone via $\mathrm{O}-\mathrm{H}$ bond insertion with water, opening an avenue to $\mathrm{CF}_{3}$ (deuteron)hydrodefluorination. ${ }^{11}$ In contrast to the aforementioned advances, $\mathrm{CF}_{3}$-activation via a difluorocarbanion intermediate remained a limited success. Although several electrochemical- and base-metal-induced protocols were reported, they usually suffered from poor chemoselectivity and limited functionalization type..$^{12}$ Meanwhile, to the best of our knowledge, enantioselective defluorofunctionalization of the $\mathrm{CF}_{3}$ group has not yet been realized. To date, the selective formation of difluorocarbanion intermediate as well as its asymmetric transformation is still a formidable challenge.

In our previous study, we have discovered that pyridine, diboron, and alkoxide could react to generate an $\mathrm{N}$-boryl pyridyl anion intermediate via heterolytic cleavage of the $\mathrm{B}-\mathrm{B}$ bond. This species is highly nucleophilic and reductive in nature, based on which a series of transformations involving redox catalysis and pyridine derivatization have been developed. ${ }^{13}$ Bearing this knowledge in mind, we sought to generate $N$-boryl pyridyl anion from 4trifluoromethylpyridine, which may undergo an in-situ fluoride elimination due to the nucleophilicity of the pyridyl anion. A subsequent alkoxide-induced deborylation 


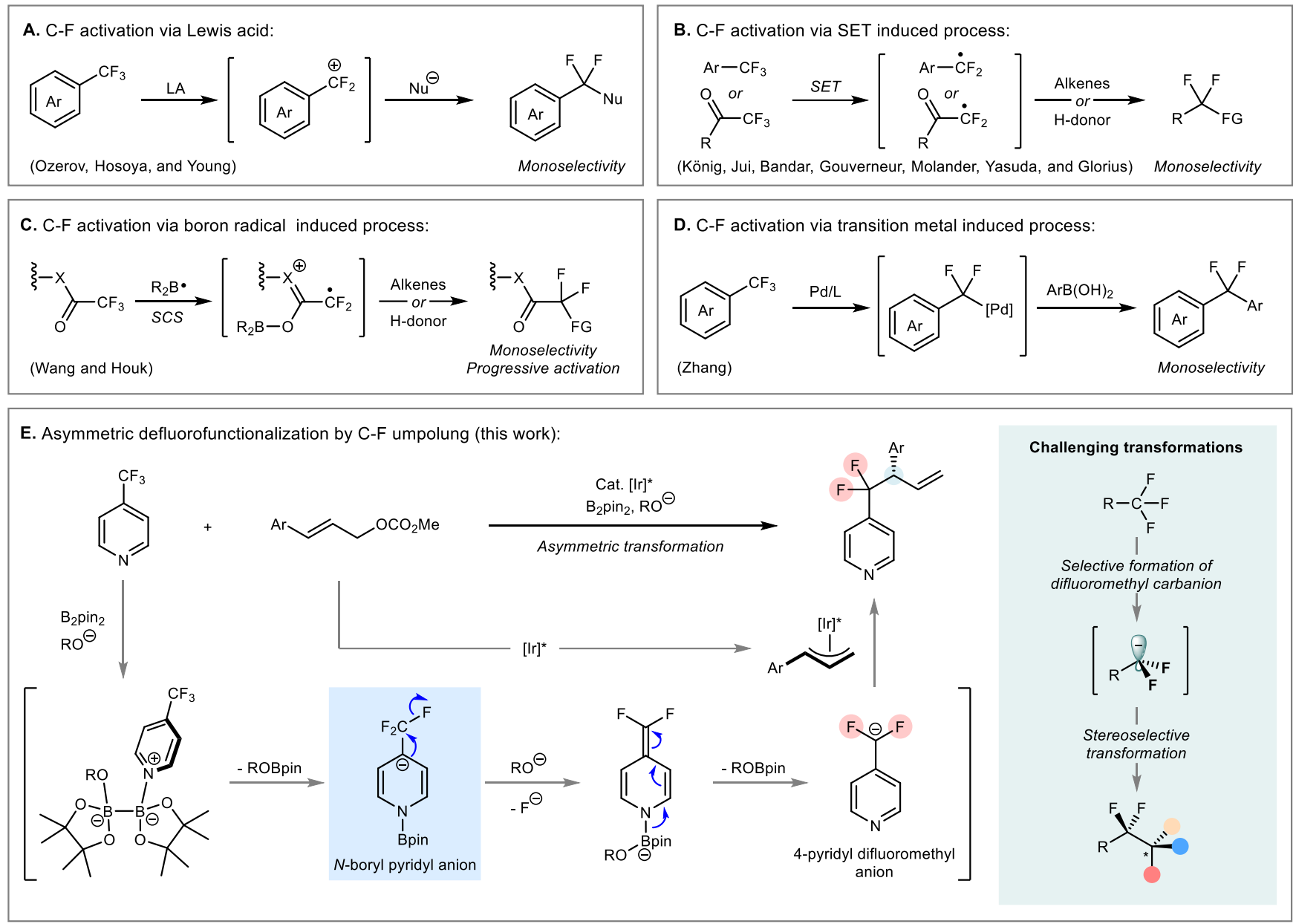

might afford a 4-pyridyldifluoromethyl anion intermediate (Scheme 1E). In this way, umpolung of the $\mathrm{CF}_{3}$ group could be achieved, enabling a new approach to fluoroalkyl anion. We further envisioned that an enantioselective transformation of this anionic species might be achieved by transition metal-catalyzed asymmetric allylic alkylation (AAA), as inspired by the successful deprotonative AAA reaction of $\alpha$-fluoro 2-pyridylacetates reported independently by Hartwig and You. ${ }^{14}$ Herein, we report the development of an Ir-catalyzed asymmetric defluoroallylation reaction of 4-trifluoropyridines, which enables the enantioselective transformation of the $\mathrm{CF}_{3}$ group via difluoromethyl anion.

To realize this design, several challenges remain to be overcome: (a) to incorporate 4-trifluoromethylpyridines into the $N$-boryl pyridyl anion chemistry; (b) to avoid decomposition of the difluoromethyl anion by alphaelimination and over-defluorination; ${ }^{15}$ and (c) to ensure the compatibility of the fluoroalkyl anion formation with the AAA reaction. Therefore, we set out to test the formation of 4-pyridyl difluoromethyl anion from 4trifluoromethylpyridine (1a) and trapping of this anion with several simple electrophiles (Scheme 2). To our delight, when benzaldehyde, paraformaldehyde, and $\mathrm{N}$ phenylbenzaldimine were employed as the electrophile, the corresponding addition products $\mathbf{2 a - c}$ were produced in good yields. Alternatively, when 10 equivalents of
$\mathrm{CD}_{3} \mathrm{OD}$ was used instead, deuterodefluorination product 2d was obtained with a high level of deuterium incorporation. These findings strongly supported the formation of the desired difluoromethyl anion intermediate or its equivalent, which served as a proof-of-concept of the designed reaction and showed that the mono defluorofunctionalization could be achieved selectively.

Scheme 2. Defluorofunctionalization of 4-Trifluoromethylpyridines
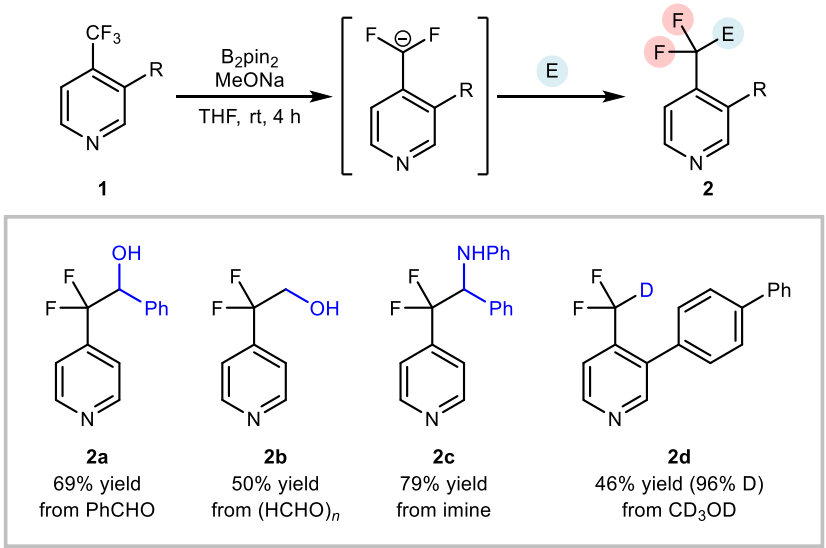
With this encouraging result in hand, we sought to merge the formation of the pyridyldifluoromethyl anion with the Ir-catalyzed AAA reaction (Table 1), although the Ir-catalyzed asymmetric defluoroallylation reaction was still unprecedented. Pyridine 1a and cinnamyl carbonate 3a were tested as template substrates. It was found that, under the optimal reaction conditions $(5 \mathrm{~mol} \%$ iridium complex $\left(S, S, S_{a}\right)$-[Ir] as the catalyst, EtONa as the base, THF as the solvent, and performing the reaction at $30{ }^{\circ} \mathrm{C}$ ), the branched defluoroallylation product $4 \mathbf{a}$ was generated in a good yield and excellent enantioselectivity (entry 1). Altering the base from EtONa to MeONa led to a lower efficiency (entry 2), and increasing or decreasing the catalyst loading resulted in diminished yields (entries 3-7). Using the insitu formed catalyst ${ }^{16}$ from ligand $\mathbf{L} 1$ and $[\operatorname{Ir}(\operatorname{cod}) \mathrm{Cl}]_{2}$ instead of the pre-formed one was proved inferior (entries 8 and 9). Other ligands (L2-L4) were found not competent for this reaction (entries 10-12).

Table 1. Optimization Study $a$

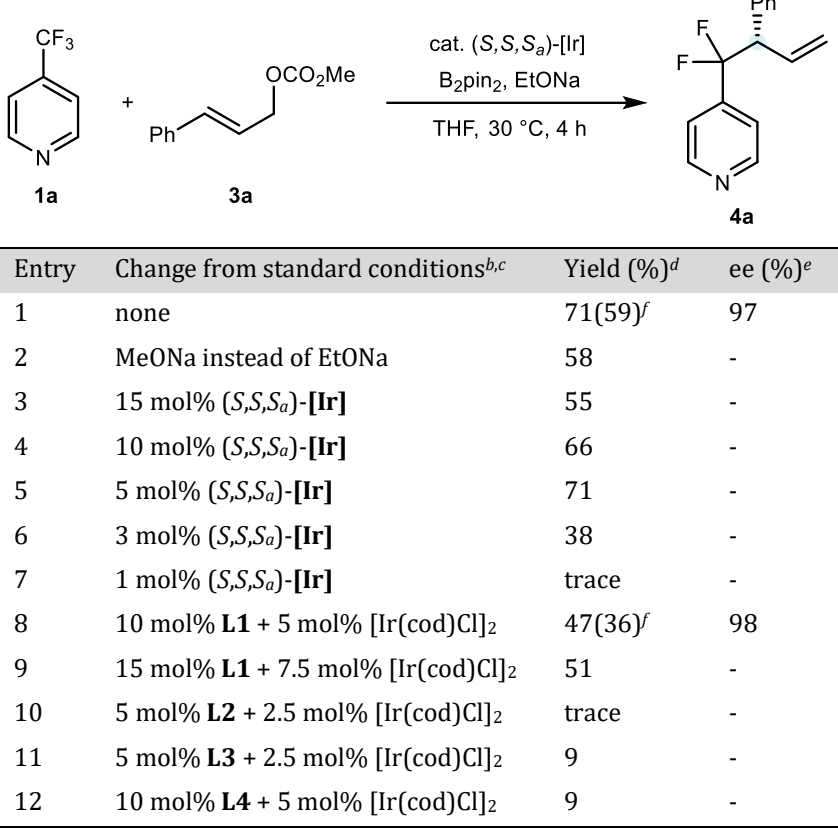

${ }^{a}$ Reaction conditions: 1a $(0.2 \mathrm{mmol}), 3 a(2.0$ equiv), EtONa (3.0 equiv), $\mathrm{B}_{2}$ pin2 (3.0 equiv), $\left(S, S, S_{a}\right)$-[Ir] (5 mol\%), THF (2 mL), Ar atmosphere, $30{ }^{\circ} \mathrm{C}, 4 \mathrm{~h} .{ }^{b} \mathrm{MeONa} \cdot \mathrm{B}_{2}$ pin 2 was used instead of EtONa and $\mathrm{B}_{2} \mathrm{pin}_{2}$ in entries 3-12. ${ }^{c}$ The Ir catalysts were pre-activated by a known preocedure for $\mathbf{L} \mathbf{1}$ L3, and L4. ${ }^{16}{ }^{d}$ Determined by ${ }^{1} \mathrm{H}$ NMR using 1,3,5-trimethoxybenzene as the internal standard. ${ }^{e}$ Determined by HPLC analysis. ${ }^{\prime}$ Yield of the isolated product.
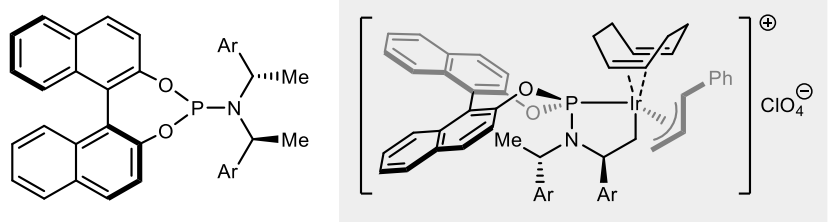

$\left(S, S, S_{a}\right)-\mathbf{L} 1(\mathrm{Ar}=4$-anisyl)

$\left(S, S, S_{a}\right)-[\operatorname{lr}](\mathrm{Ar}=4$-anisyl)

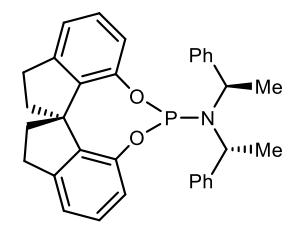

$\left(R, R, R_{a}\right)-\mathrm{L} 2$

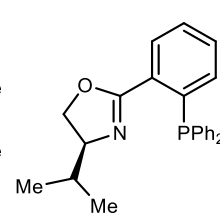

(S)-L3

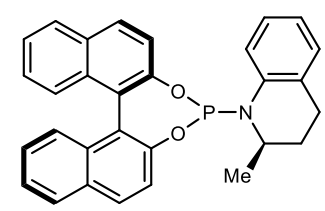

$\left(R, R_{a}\right)-\mathrm{L} 4$
With the optimal reaction conditions in hand, we started to explore the scope of this asymmetric difluoroallylation reaction (Table 2). The reactions of 4-trifluoropyridine (1a) with various allyl carbonates were first examined. The cinnamyl carbonates bearing electron donating groups on the phenyl ring were suitable reactants, delivering the target products $\mathbf{4 b - d}$ in good yields and excellent enantioselectivities. 3,4,5-Trimethoxy- and 3-methylsubstituted cinnamyl carbonates afforded the corresponding products $4 \mathbf{e}$ and $\mathbf{4 f}$ in moderate yields with excellent enantioselectivities. 4-Halogen substituents on the phenyl ring of the cinnamyl carbonate were tolerated in this reaction, albeit with diminished yield and selectivity. Allyl carbonates bearing other aromatic rings, such as thiophene and naphthalene, were also compatible substrates to afford $4 \mathbf{i}$ and $\mathbf{4 j}$ in moderate yields and excellent selectivities. It is noteworthy that an ortho-substitution on the phenyl group led to a decreased enantioselectivity (4k). It was found that, alkyl substituted allylic carbonate was not a suitable substrate, which exhibited greatly diminished reactivity.

Subsequently, the scope of the fluoroalkyl anion source was examined (Table 2). 4-Trifluoromethylpyridines bearing various 3-substituents were found compatible for this defluoroallylation reaction. Aryl groups of different electronic properties were tolerated (4l-4o), and in particular, another pyridine core remained intact during the reaction (4p). Further investigation showed that 3-vinyl and benzyl substituted 4-trifluoromethylpyridines also behaved well to furnish $\mathbf{4 q}$ and $\mathbf{4 r}$ in good yields and enantioselectivity. Notably, 4-trifluoromethylpyrimidines also underwent the desired reaction smoothly under identical reaction conditions to give a satisfactory outcome $4 \mathbf{4 s}$ and 4t), exhibiting the potential of the present method for adapting other N-heterocycles. Pyridine substrates bearing electron-withdrawing substitutions at the 3-position participated in the reaction smoothly to afford the corresponding defluoroalkylation products $\mathbf{4 u - 4 z}$ in high yields and excellent selectivities, among which functional groups such as ester, amide, tertary amine, and geraniol ester were well tolerated. The present protocol also allowed for reaction at a larger scale without deterioration of yield and enantioselectivity, as demonstrated by $2 \mathrm{mmol}$ scale synthesis of $\mathbf{4 c}$.

As for the limitation of this reaction, we found that both 2-substituted 4-trifluoromethylpyridine and 2-trifluoromethylpyridine exhibited no reactivity, implying that the formation of the $N$-boryl pyridyl anion intermediate is sensitive to steric hindrance.

To explore the synthetic utility of this reaction, we investigated the derivatization of the defluoroallylation products (Scheme 3). First, derivatization of the vinyl group was attempted, which could be transformed to a hydroxyethylene group by hydroboration-oxidation sequence to produce alcohol 5 in $71 \%$ yield and $97 \%$ ee. Ozonation of the double bond followed by reduction furnished the alcohol product 6 in 52\% yield and $98 \%$ ee. Second, we tested whether a second defluorofunctionalization could be achieved by applying the $N$-boryl pyridyl anion chemistry. Gratifyingly, it was found that product 4c could undergo defluorination and nucleophilic addition with 2- 

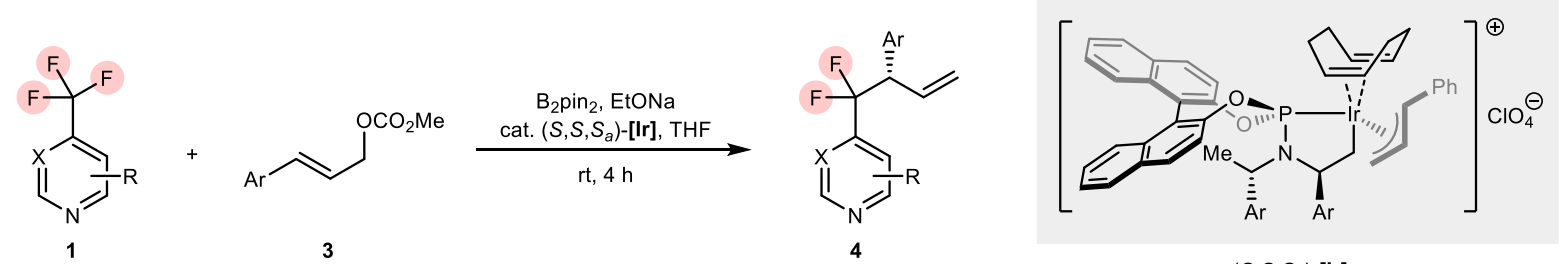

$\left(S, S, S_{a}\right)-[\operatorname{lr}]$

Scope of allyl carbonate

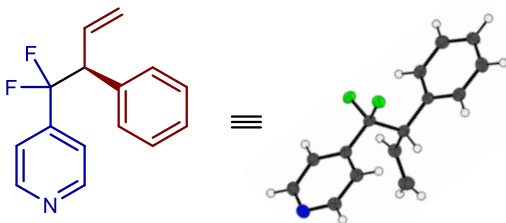

4a
$59 \%$ yield, $97 \%$ ee<smiles>C=C[C@@H](c1cccc(C)c1)C(F)(F)c1ccncc1</smiles>

$53 \%$ yield, $97 \%$ ee
XRD of (S)-4a<smiles>C=C[C@@H](c1ccc(F)cc1)C(F)(F)c1ccncc1</smiles>

57\% yield, $97 \%$ ee

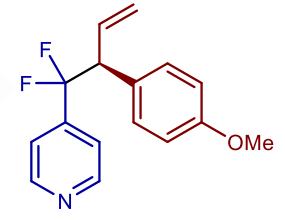

4b

$81 \%$ yield, $99 \%$ ee

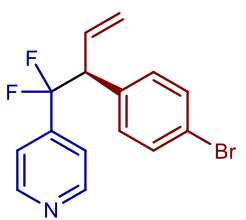

$\stackrel{\text { 4h }}{37 \% \text { yield, } 92 \% \text { ee }}$<smiles>C=CC(c1ccc(C)cc1)C(F)(C=C)C(F)(F)c1ccncc1</smiles>

4d $\mathbf{4 c}$
$80 \%$ yield, $98 \%$ ee
$[76 \% \text { yield, } 98 \% \text { ee }]^{b}$<smiles>C=C[C@@H](c1cccs1)C(F)(F)c1ccncc1</smiles>

57\% yield, $>\mathbf{4 i}$ ee

$\stackrel{4 j}{50 \% \text { yield, } 97 \% \text { ee }}$

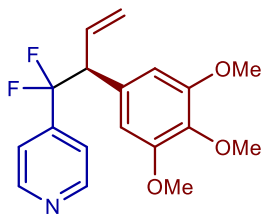

$4 \mathrm{e}$

$45 \%$ yield, $97 \%$ ee

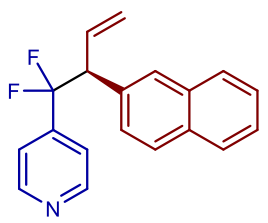

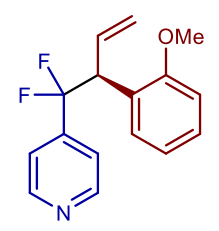

$4 \mathrm{k}$

$80 \%$ yield, $85 \%$ ee

Scope of trifluoromethylpyridine<smiles>C=C[C@@H](Cl)C(F)(F)c1ccncc1-c1ccc(-c2ccccc2)cc1</smiles>

41

$79 \%$ yield, $98 \%$ ee<smiles>C=C[C@@H](C)C(F)(F)c1ccncc1-c1ccc(N2CCOCC2)nc1</smiles>

$4 p$
$77 \%$ yield, $96 \%$ ee<smiles>C=C[C@@H](Br)C(F)(F)c1ccncc1C(=O)OCC</smiles>

$\mathrm{Ar}=\mathrm{Tol}, \mathbf{4 u}, \mathbf{7 3} \%$ yield, $97 \%$ ee $\mathrm{Ar}=$ Anisyl, $\mathbf{4 v}, 75 \%$ yield, $97 \%$ ee<smiles>C=C[C@@H]([AlH2])C(F)(F)c1ccncc1-c1ccc2ccccc2c1</smiles>

$\stackrel{4 m}{82 \% \text { yield, }>99 \% \text { ee }}$<smiles>C=C[C@H](Cl)C(F)(F)c1ccncc1-c1ccccc1</smiles>

$4 \mathrm{n}$

$85 \%$ yield, $98 \%$ ee<smiles>C=C[C@@H](Cl)C(F)(F)c1ccncc1-c1ccoc1</smiles>

40

$80 \%$ yield, $98 \%$ ee

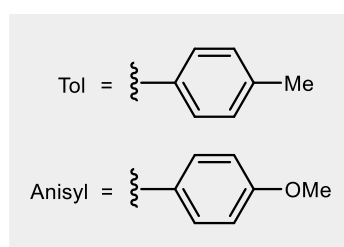

(1)

$4 \mathrm{~s}$

$55 \%$ yield, $97 \%$ ee<smiles>C=C[C@@H]([AlH2])C(F)(F)c1ncncc1-c1ccc2c(c1)OCO2</smiles>
$59 \%$ yield, $97 \%$ ee

${ }^{a}$ Reaction conditions: 1 (0.5 mmol, 1 equiv), 3 (1.0 mmol, 2 equiv), B $\mathrm{B}_{2} \mathrm{pin}_{2}$ (1.5 mmol, 3 equiv), EtONa (1.5 mmol, 3 equiv) and $\left(S, S, S_{a}\right)$-[Ir] $(0.025 \mathrm{mmol}, 5 \mathrm{~mol} \%)$ in $5 \mathrm{~mL}$ of THF, Ar atmosphere, $30^{\circ} \mathrm{C}, 4 \mathrm{~h} .{ }^{b}$ Reaction was carried out on $2.0 \mathrm{mmol}$ scale.

phenylbenzaldehyde under slightly more forcing reaction conditions, and the addition product could be oxidized to afford ketone 7 in 38\% overall yield and 2:1 dr. Meanwhile, cinnamyl chloride could also be employed as the electro- phile in this transformation to produce allylated product 8 in 2:1 dr, which afforded cyclized product 9 after ringclosing olefin metathesis to serve as an indicator for stereochemistry. Although the yield and diastereoselectivity 
was not perfect, these results showed that sequential monoselective $\mathrm{C}-\mathrm{F}$ activation of 4-trifluoropyridine is viable with the present protocol.

\section{Scheme 3. Derivatization of Products ${ }^{a}$}

A. Derivatization of the vinyl group:

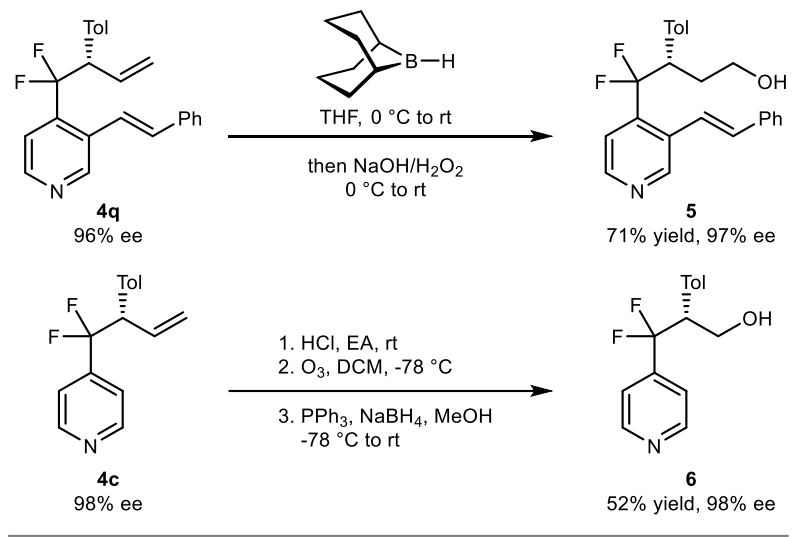

B. Second $\mathrm{C}-\mathrm{F}$ bond functionalization:

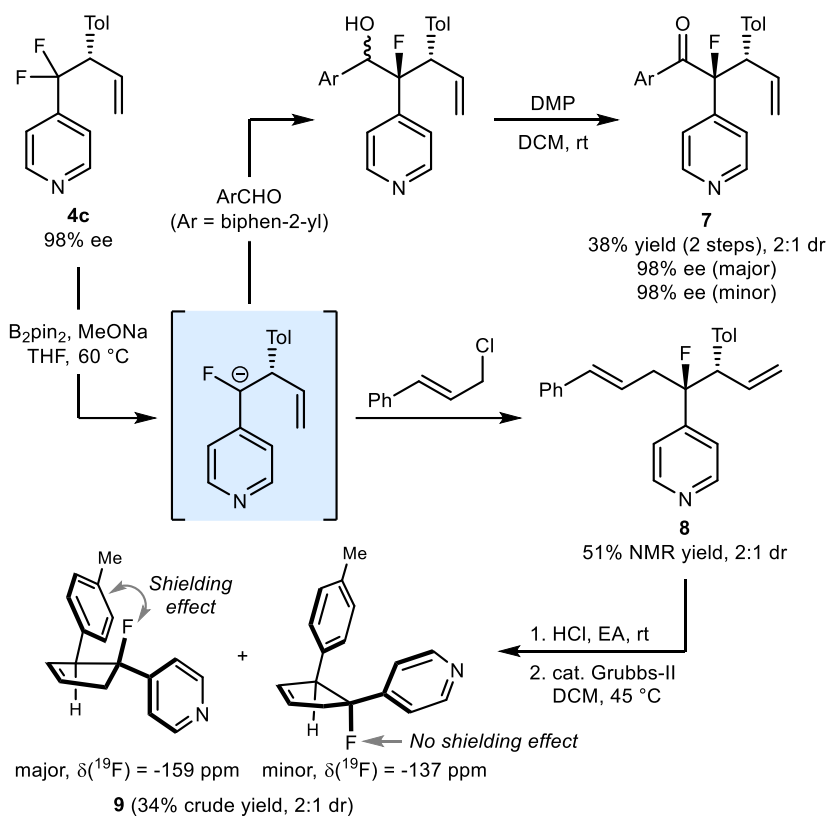

${ }^{a} \mathrm{DMP}=$ Dess-Martin periodane; DCM = dichloromethane, $\mathrm{EA}=$ ethyl acetate; $\mathrm{rt}=$ room temperature

Interestingly, in addition to trifluoromethylpyridines, methyl pyrid-4-ylmethyl ethers were also compatible for this umpolung process. Ethers $\mathbf{1 0}$ and $\mathbf{1 1}$ were found to undergo a demethoxyalkylation reaction smoothly with alkyl halides under the established conditions to afford the alkylated products $\mathbf{1 2}$ and $\mathbf{1 3}$, albeit an AAA reaction was not successful at the moment. Nevertheless, this result indicated that the $N$-boryl pyridyl anion chemistry could serve as a generic strategy for umpolung of the pyrid-4ylmethyl motif bearing a leaving group to enable its nucleophilic reactivity.
Scheme 4. Demethoxylalkylation reactions

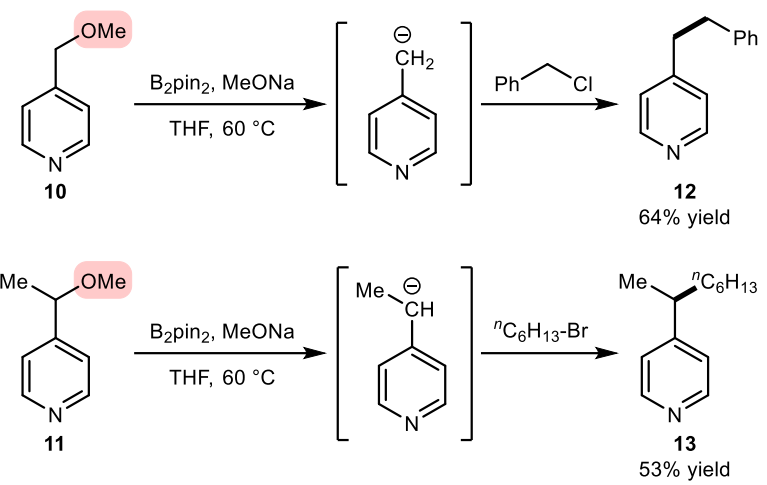

In conclusion, we have developed a monoselective defluorofunctionalization reaction of 4-trifluoromethylpyridines by invoking the diboron-base-pyridine system. This protocol leads to the umpolung of the fluoroalkyl unit on the pyridine ring, and allows for an asymmetric transformation of the $\mathrm{CF}_{3}$ group. The present work opens up a new opportunity for the functionalization of trifluoromethylpyridines, and provides new insights into the $\mathrm{N}$ boryl pyridyl anion chemistry. We expect this reaction system to become a versatile platform for the umpolung and functionalization of pyridylmethyl moiety, and the corresponding studies are ongoing.

\section{ASSOCIATED CONTENT}

Supporting Information. Experimental procedures, characterization data, and copies of NMR spectra.

\section{AUTHOR INFORMATION}

\section{Corresponding Author}

Lei Jiao - Center of Basic Molecular Science, Department of Chemistry, Tsinghua University, Beijing 10084, China, leijiao@mail.tsinghua.edu.cn

\section{Funding Sources}

Financial support was provided by the National Natural Science Foundation of China (grant number 21822304 to L.J.).

Notes

The authors declare no competing financial interest.

\section{ACKNOWLEDGMENT}

The technological platform of CBMS is acknowledged for providing instrumentation.

\section{REFERENCES}

(1) Smart, B. E. Fluorine substituent effects (on bioactivity). J. Fluorine Chem. 2001, 109, 3.

(2) (a) Müller, K.; Faeh, C.; Diederich, F. Fluorine in pharmaceuticals: Looking beyond intuition. Science 2007, 317, 1881. (b) Hagmann, W. K. The many roles for fluorine in medicinal chemistry. J. Med. Chem. 2008, 51, 4359. (c) Zhou, Y.; Wang, J.; Gu, Z.; Wang, S.; Zhu, W.; Aceña, J. L.; Soloshonok, V. A.; Izawa, K.; Liu, H. Next Generation of Fluorine-Containing Pharmaceuticals, Compounds Currently in Phase II-III Clinical Trials of Major Pharmaceutical Companies: New Structural Trends and Therapeutic Areas. Chem. Rev. 2016, 116, 422. 
(3) (a) Liang, T.; Neumann, C. N.; Ritter, T. Introduction of Fluorine and Fluorine-Containing Functional Groups. Angew. Chem. Int. Ed. 2013, 52, 8214. (b) Barata-Vallejo, S.; Lantaño, B.; Postigo, A. Recent Advances in Trifluoromethylation Reactions with Electrophilic Trifluoromethylating Reagents. Chem. - Eur. J. 2014, 20, 16806. (c) Alonso, C.; de Marigorta, E. M.; Rubiales, G.; Palacios, F. Carbon Trifluoromethylation Reactions of Hydrocarbon Derivatives and Heteroarenes. Chem. Rev. 2015, 115, 1847.

(4) (a) Cho, E. J.; Senecal, T. D.; Kinzel, T.; Zhang, Y.; Watson, D. A.; Buchwald, S. L. The Palladium-Catalyzed Trifluoromethylation of Aryl Chlorides. Science 2010, 328, 1679. (b) Nagase, M.; Kuninobu, Y.; Kanai, M. 4-Position-Selective C-H Perfluoroalkylation and Perfluoroarylation of Six-Membered Heteroaromatic Compounds. J. Am. Chem. Soc. 2016, 138, 6103. (c) Ye, F.; Berger, F.; Jia, H.; Ford, J.; Wortman, A.; Börgel, J.; Genicot, C.; Ritter, T. Aryl Sulfonium Salts for Site-Selective Late-Stage Trifluoromethylation. Angew. Chem. Int. Ed. 2019, 58, 14615. (d) Zhang, X.; Nottingham, K. G.; Patel, C.; Alegre-Requena, J. V.; Levy, J. N.; Paton, R. S.; McNally, A. Phosphorus-mediated $\mathrm{sp}^{2}-\mathrm{sp}^{3}$ couplings for $\mathrm{C}-\mathrm{H}$ fluoroalkylation of azines. Nature 2021, 594, 217.

(5) (a) Amii, H.; Uneyama, K. C-F Bond Activation in Organic Synthesis. Chem. Rev. 2009, 109, 2119. (b) Shen, Q.; Huang, Y.-G.; Liu, C.; Xiao, J.-C.; Chen, Q.-Y.; Guo, Y. Review of recent advances in $\mathrm{C}-\mathrm{F}$ bond activation of aliphatic fluorides. J. Fluorine Chem. 2015, 179, 14. (c) Hamel, J.-D.; Paquin, J.-F. Activation of C-F bonds $\alpha$ to C-C multiple bonds. Chem. Commun. 2018, 54, 10224. (d) Ai, H.-J.; $\mathrm{Ma}, \mathrm{X}$.; Song, Q.; Wu, X.-F. C-F bond activation under transitionmetal-free conditions. Sci. China Chem. 2021, 64, 1630.

(6) (a) Ma, X.; Song, Q. Recent progress on selective deconstructive modes of halodifluoromethyl and trifluoromethylcontaining reagents. Chem. Soc. Rev. 2020, 49, 9197. (b) Zhao, F.; Zhou, W.; Zuo, Z. Recent Advances in the Synthesis of Difluorinated Architectures from Trifluoromethyl Groups. Adv. Synth. Catal. 2021, DOI: 10.1002/adsc.202101234.

(7) (a) Douvris, C.; Ozerov, 0. V. Hydrodefluorination of perfluoroalkyl groups using silylium-carborane catalysts. Science 2008, 321, 1188. (b) Yoshida, S.; Shimomori, K.; Kim, Y.; Hosoya, T. Single C-F Bond Cleavage of Trifluoromethylarenes with an orthoSilyl Group. Angew. Chem. Int. Ed. 2016, 55, 10406. (c) Mandal, D.; Gupta, R.; Jaiswal, A. K.; Young, R. D. Frustrated Lewis-PairMeditated Selective Single Fluoride Substitution in Trifluoromethyl Groups. J. Am. Chem. Soc. 2020, 142, 2572.

(8) (a) Chen, K.; Berg, N.; Gschwind, R.; König, B. Selective Single $\mathrm{C}\left(\mathrm{sp}^{3}\right)-\mathrm{F}$ Bond Cleavage in Trifluoromethylarenes: Merging Visible-Light Catalysis with Lewis Acid Activation. J. Am. Chem. Soc. 2017, 139, 18444. (b) Wang, H.; Jui, N. T. Catalytic Defluoroalkylation of Trifluoromethylaromatics with Unactivated Alkenes. J. Am. Chem. Soc. 2018, 140, 163. (c) Luo, C.; Bandar, J. S. Selective Defluoroallylation of Trifluoromethylarenes. J. Am. Chem. Soc. 2019, 141, 14120. (d) Vogt, D. B.; Seath, C. P.; Wang, H.; Jui, N. T. Selective C-F Functionalization of Unactivated Trifluoromethylarenes. J. Am. Chem. Soc. 2019, 141, 13203. (e) Sap, J. B. I.; Straathof, N. J. W.; Knauber, T.; Meyer, C. F.; Médebielle, M.; Buglioni, L.; Genicot, C.; Trabanco, A. A.; Noël, T.; Ende, C. W. A.; Gouverneur, V. Organophotoredox Hydrodefluorination of Trifluoromethylarenes with Translational Applicability to Drug Discovery. J. Am. Chem. Soc. 2020, 142, 9181. (f) Campbell, M. W.; Polites, V. C.; Patel, S.; Lipson, J. E.; Majhi, J.; Molander, G. A. Photochemical C-F Activation Enables Defluorinative Alkylation of Trifluoroacetates and -Acetamides. J. Am. Chem. Soc. 2021, 143, 19648. (g) Sugihara, N.; Suzuki, K.; Nishimoto, Y.; Yasuda, M. Photoredox-Catalyzed C-F Bond Allylation of Perfluoroalkylarenes at the Benzylic Position. J. Am. Chem. Soc. 2021, 143, 9308. (h) Ye, J.-H.; Bellotti, P.; Heusel, C.; Glorius, F. Photoredox-Catalyzed Defluorinative Functionalizations of Polyfluorinated Aliphatic Amides and Esters. Angew. Chem. Int. Ed. 2021, DOI: 10.1002 /anie.202115456.
(9) Yu, Y.-J.; Zhang, F.-L.; Peng, T.-Y.; Wang, C.-L.; Cheng, J.; Chen, C.; Houk, K. N.; Wang, Y.-F. Sequential C-F bond functionalizations of trifluoroacetamides and acetates via spin-center shifts. Science 2021, 371, 1232.

(10) Luo, Y.-C.; Tong, F.-F.; Zhang, Y.; He, C.-Y.; Zhang, X. VisibleLight-Induced Palladium-Catalyzed Selective Defluoroarylation of Trifluoromethylarenes with Arylboronic Acids. J. Am. Chem. Soc. 2021, 143, 13971.

(11) Zhang, X.; Zhang, X.; Song, Q.; Sivaguru, P.; Wang, Z.; Zanoni, G.; Bi, X. A Carbene Strategy for Progressive (Deutero)Hydrodefluorination of Fluoroalkyl Ketones. Angew. Chem. Int. Ed. 2021, DOI: 10.1002/anie.202116190.

(12) (a) Saboureau, C.; Troupel, M.; Sibille, S.; Périchon, J. Electroreductive Coupling of Trifluoromethylarenes with Electrophiles: Synthetic Applications. J. Chem. Soc., Chem. Comm. 1989, 1138. (b) Clavel, P.; Léger-Lambert, M.-P.; Biran, C.; SereinSpirau, F.; Bordeau, M.; Roques, N.; Marzouk, H. Selective electrosynthesis of (trimethylsilyldifluoro)methylbenzene, a $\mathrm{PhCF}_{2}^{-}$precursor; conditions for a molar scale preparation without HMPA. Synthesis 1999, 829. (c) Amii, H.; Hatamoto, Y.; Seo, M.; Uneyama, K. New C-F bond-cleavage route for the synthesis of octafluoro[2.2] paracyclophane. J. Org. Chem. 2001, 66, 7216. (d) Clavel, P.; Lessene, G.; Biran, C.; Bordeau, M.; Roques, N.; Trévin, S.; de Montauzon, D. Selective electrochemical synthesis and reactivity of functional benzylic fluorosilylsynthons. J. Fluorine Chem. 2001, 107, 301. (e) Yamauchi, Y.; Fukuhara, T.; Hara, S.; Senboku, H. Electrochemical carboxylation of $\alpha, \alpha-$ difluorotoluene derivatives and its application to the synthesis of $\alpha$-fluorinated nonsteroidal anti-inflammatory drugs. Synlett 2008, 438. (f) Utsumi, S.; Katagiri, T.; Uneyama, K. Cu-deposits on Mg metal surfaces promote electron transfer reactions. Tetrahedron 2012, 68, 1085. (g) Munoz, S. B.; Ni, C.; Zhang, Z.; Wang, F.; Shao, N.; Mathew, T.; Olah, G. A.; Prakash, G. K. S. Selective Late-Stage Hydrodefluorination of Trifluoromethylarenes: A Facile Access to Difluoromethylarenes. Eur. J. Org. Chem. 2017, 2017, 2322.

(13) (a) Zhang, L.; Jiao, L. Pyridine-Catalyzed Radical Borylation of Aryl Halides. J. Am. Chem. Soc. 2017, 139, 607. (b) Zhang, L.; Jiao, L. Super electron donors derived from diboron. Chemical Science 2018, 9, 2711. (c) Zhang, L.; Jiao, L. Visible-Light-Induced Organocatalytic Borylation of Aryl Chlorides. J. Am. Chem. Soc. 2019, 141, 9124. (d) Yang, H.; Zhang, L.; Zhou, F.-Y.; Jiao, L. An umpolung approach to the hydroboration of pyridines: a novel and efficient synthesis of $\mathrm{N}$-H 1,4-dihydropyridines. Chemical Science 2020, 11, 742. (e) Zhang, L.; Wu, Z.-Q.; Jiao, L. Photoinduced Radical Borylation of Alkyl Bromides Catalyzed by 4-Phenylpyridine. Angew. Chem. Int. Ed. 2020, 59, 2095.

(14) (a) He, Z.-T.; Jiang, X.; Hartwig, J. F. Stereodivergent Construction of Tertiary Fluorides in Vicinal Stereogenic Pairs by Allylic Substitution with Iridium and Copper Catalysts. J. Am. Chem. Soc. 2019, 141, 13066. (b) Liu, X.-J.; Jin, S.; Zhang, W.-Y.; Liu, Q.-Q.; Zheng, C.; You, S.-L. Sequence-Dependent Stereodivergent Allylic Alkylation/Fluorination of Acyclic Ketones. Angew. Chem. Int. Ed. 2020, 59, 2039.

(15) Santos, L.; Panossian, A.; Donnard, M.; Vors, J.-P.; Pazenok, S.; Bernier, D.; Leroux, F. R. Deprotonative Functionalization of the Difluoromethyl Group. Org. Lett. 2020, 22, 8741.

(16) Hartwig, J. F.; Stanley, L. M. Mechanistically Driven Development of Iridium Catalysts for Asymmetric Allylic Substitution. Acc. Chem. Res. 2010, 43, 1461. 


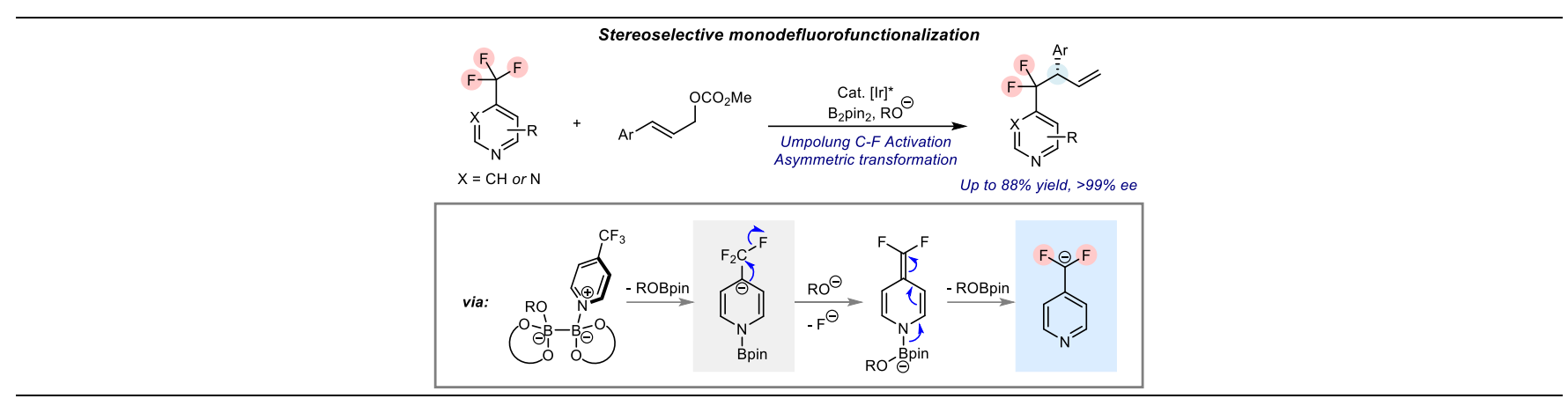

